\title{
Editorial
}

\section{The HIV/SIV Envelope Protein: From Structure To Function To Host Evasion}

It is a pleasure to write an introduction for this collection of five short reviews of primate lentivirus Env proteins. These are very timely reviews, each designed to cover a different aspect of the biology of these proteins. This is a remarkable time to organize what we know about this family of proteins. There has been a long-standing interest in these proteins for their role in viral entry. The Env protein is the only viral protein on the surface of the virion making it the sole protein involved in entry. The synthesis of the Env protein is somewhat unremarkable, being a typical type 1 transmembrane protein synthesized as a large precursor on the rough ER. There is the co-translational addition of carbohydrate within the ER and trimerization of the Env protein precursor. Then in the golgi, a host protease cleaves the larger precursor into an extracellular surface protein (SU subunit) and a transmembrane protein (TM subunit), creating a new $\mathrm{N}$ terminus of the TM protein that functions as the fusion peptide in the fusion event. Despite this cleavage event, the SU and TM proteins remain together, with three of each subunit comprising an Env trimer.

Two unusual features of Env biology can be observed in mature virions. First, Env is one of the most heavily glycosylated proteins known. Second, the density of the Env protein trimer on the surface of the virion is very low. Both of these features probably speak to the fact that with the virus undergoing constant replication, there must be constant evasion from the host antibody response. The high density of glycans blocks binding of antibodies to the otherwise immunogenic surface of the protein, while the low density of trimers on the virion surface reduces the ability of bi-functional antibodies to engage two separate trimers and overall reduces the amount of antigen on the virion.

Our reviews start with Env on the virus particle. This is a restless protein, being able to exist in multiple conformations. Important insights into the structure of the Env trimer have become available in recent years using cryoEM and the constrained SOSIP form of the protein, with a review by Ward and Wilson being currently available as open access (https://www.ncbi.nlm.nih.gov/pmc/articles/PMC4310573/). However, as the saying goes "No one ever learned how to dance by looking at pictures." Kelly Lee and James Munro work on methods that build on static models of Env structure and allow an assessment of the dynamics and conformational changes in the protein. Their review describes approaches that will continue to provide novel insights into how the Env protein can exist in several distinct states, either during transition to membrane fusion or after selection for different biological properties.

The Env protein on the virus particle is the immunogen for the development of neutralizing antibodies by the host. Much attention has been paid in the last few years to the development of broadly neutralizing antibodies within a small number of people. A current open access review describing the methodology for identifying such antibodies and their targets on the otherwise well-defended Env protein was written by McCoy and Burton (https:/www.ncbi.nlm.nih.gov/pubmed/28133814). For our series, we asked Penny Moore to take a step back and review the more general ontogeny of the neutralizing antibody response to HIV-1 in its human host and how that response can, on occasion, expand beyond the type-specific response to the virus in that host to give a more broad neutralizing response.

The remaining three reviews deal with the specificity of virus entry. The broad details of the entry process are well known, with CD4 as the primary receptor and a chemokine receptor as a secondary or coreceptor, with CCR5 most often serving this role. The sequential binding of the receptor and coreceptor induces a series of conformational changes that result in fusion of the viral envelope membrane and the host cell plasma membrane. Each of these steps can be subjected to selective pressure that results in functional changes, with selection occurring naturally during the course of infection or artificially at the hands of clever investigators in the laboratory. In one review, Council and Joseph discuss the host selective pressures driving the evolution of HIV-1 entry phenotypes. They remind us that the normal form of HIV-1 uses CCR5 and requires a high density of CD4 to enter cells (as found on CD4+ T cells), but these phenotypes can evolve and change, usually late in disease. First, there is the well known coreceptor switch where the virus gives up CCR5 for CXCR4 as its coreceptor. Second, and less well appreciated, the virus can evolve the ability to enter cells that have a low density of CD4, such as macrophages. They also review reports of alternative pathways of targeting and entering cells.

The review by Swanstrom, Hoxie and colleagues moves this discussion to SIV infection of macaques. There are many parallels in the biology of HIV-1 entry phenotypes in people and infection of rhesus macaques with SIV strains derived from infected sooty mangabeys (SIVsm or SIVmac when adapted to macaques). In addition, the use of an animal model allows greater assessment of virus throughout the body. Finally, manipulations of the Env protein can be evaluated for alterations in pathogenesis and targeting within a primate host. All of these issues are discussed in this wide-ranging review. 
In the final review of this group, Wetzel, Elliott, and Collman examine the evidence that plasticity in the use of coreceptor goes well beyond the CCR5 to CXCR4 switch known for HIV-1. When one looks across the natural SIV infections that occur in old world monkeys, issues of alternative coreceptor usage become apparent. Alternative coreceptors likely represent coevolution between the host and the virus that results in a persistent, nonpathogenic infection, a surprising and important insight given the extreme pathogenesis associated with HIV-1 infection in humans.

In these reviews we have tried to engage younger researchers (sometimes with their mentors) to give them an opportunity to profile their own work and to place it in the context of the broader field. It has been a pleasure to see this collection of complementary reviews come together. We are especially pleased that these reviews are available through open access to make them as useful to the community as possible. We hope you find them insightful and that they contribute to your understanding of the viral Env protein in its role in entry and as a determinant of pathogenesis.

\section{Ronald Swanstrom}

Sarah B. Joseph

\section{(Guest Editor)}

Department of Biochemistry and Biophysics

University of North Carolina at Chapel Hill

Chapel Hill, NC

USA

E-mail: risunc@med.unc.edu

\section{(Co-Guest Editor)}

Department of Microbiology and Immunology University of North Carolina at Chapel Hill

Chapel Hill, NC

USA 